\title{
STH - Sistema Teleatendimento Holográfico: Um sistema de videoconferência para o Sistema de Saúde Holográfico da UFF
}

\author{
André Luis de Oliveira Fonseca ${ }^{1}$, Natalia Castro Fernandes ${ }^{1}$ e \\ Ricardo Campanha Carrano ${ }^{1}$
}

\author{
${ }^{1}$ Laboratório Midiacom - Escola de Engenharia \\ Universidade Federal Fluminense (UFF) \\ Rua Passo da Pátria, 156 - São Domingos - Niterói - RJ - CEP 24210-240
}

\begin{abstract}
This article presents a control and managed tool for videoconferencing telepresence system for medical teleinterconsultations with holography. The main focus of the system is specialized medical care at remote locations through bidirectional audio and video streaming, whether by physical cable or by satellite, depending on an available infrastructure. The tool developed is adapted to the environment with holographic projection and friendly for the complete usability of the system by the health teams. With its modular architecture, it allows the insertion of new medical sensors in a simple way, besides complying with the requirements imposed by the legislation, regarding the transmission and the safe storage of the data. The system was tested with different medical specialties and was very well accepted by health professionals.
\end{abstract}

Resumo. Este artigo apresenta a ferramenta de controle e gerência desenvolvida para o sistema de telepresença por videocolaboração para teleinterconsultas médicas com holografia da UFF. O principal foco do sistema é prover atendimento médico especializado a locais remotos através de transmissão bidirecional com streaming de áudio e vídeo, seja meio físico terrestre ou via satélite, dependendo da infra-estrutura disponível. A ferramenta desenvolvida é adaptada ao ambiente com projeção holográfica e amigável para a completa operação do sistema pelas equipes de saúde. Com sua arquitetura modular, permite a inserção de novos sensores médicos de forma simples, além de atender aos requisitos impostos pela legislação, com relação à transmissão e ao armazenamento seguro dos dados. O sistema foi testado com diferentes especialidades médicas, tendo sido muito bem aceito pelos profissionais de saúde.

\section{Introdução}

A falta de médicos em determinados pontos do nosso pais deixa a população sem atendimento especializado, fazendo com que boa parte sofra sem um atendimento adequado ao seu quadro clínico [Scheffer et al. 2018]. Levando em conta esse cenário, foi proposto o projeto de Telessaúde com Holografia da UFF [Fonseca et al. 2017]. O objetivo principal do projeto é prover atendimento especializado a locais remotos, visando inicialmente atender a população do interior do Brasil onde o atendimento de saúde é precário, em especial na Amazônia.

A proposta do ambiente é trazer a sensação de imersão de um atendimento real para os médicos que estão auxiliando a consulta em um ponto geograficamente remoto, 
levando a sensação que o paciente esteja no mesmo local que a junta médica especializada [FAPERJ 2017]. Sendo assim, os médicos podem perceber movimentos, reações e outros fatores relacionados ao paciente que possam auxiliar o diagnóstico ou orientar o profissional no ambiente de atendimento remoto. O sistema de atendimento consiste em dois ambientes: o Centro de Saúde Holográfico (CSH), que é o local aonde os especialistas observam o paciente holograficamente, e o Consultório de Saúde Virtual (CSV), que é o consultório na localidade remota [Fonseca et al. 2017].

O SSH-UFF possui interação manual de comunicação via streaming de áudio e vídeo utilizado para comunicação entre o CSV e CSH [Dal Bello 2016]. Existe a necessidade de suporte técnico para auxiliar os médicos durante a teleconsulta . Contudo precisa-se de uma ferramenta que auxilia de forma amigável as configurações para que não somente um operador possa utilizado-o, deixando o profissional de saúde ou o médico com a liberdade de interação sem a necessidade do suporte técnico. Para que o ambiente se tornasse realidade, foi desenvolvido, em código aberto, uma ferramenta de gestão e controle para viabilizar e tornar amigável a gerência e a operação do Sistema de Saúde Holográfico ${ }^{1}$.

A ferramenta proposta é modular, dando auxílio a inserção de diferentes tipos de imagens e sensores médicos no sistema, além de dar suporte às exigências das regulamentações médicas. A ferramenta desenvolvida permite a criação de uma conexão segura para a videoconferência. Toda a comunicação é bidirecional com streaming de áudio e vídeo, considerando a necessidade de prover um vídeo e um áudio de qualidade mesmo quando as condições da rede são uma baixa taxa de dados com atraso, o que é comum nas áreas aonde o sistema é aplicado.

\section{Trabalhos relacionados}

Dado o amplo foco que vem sendo dado à telessaúde atualmente, alguns softwares foram desenvolvidos. Entre os sistemas abertos, destacam-se a iniciativa Rede RUTE [RUTE 2019] e o sistema desenvolvido pela UFRGS [Roesler et al. 2015].

A Rede RUTE é uma rede colaborativa de pesquisa que conecta em alta velocidade 136 unidades em operação em todo o Brasil, com 50 Grupos de Interesse Especial (SIGs) em 12 anos de atuação [RUTE 2019]. A Rede RUTE conecta e integra todos os hospitais públicos universitários e cria formalmente unidades de telemedicina estimulando assistência, ensino, pesquisa, monitoramento, gestão e avaliação remota para área da saúde, utilizando a infra-estatura da Rede Nacional de Ensino e Pesquisa (RNP).

O sistema Multipresença [Roesler et al. 2015] [Roesler and Harzheim 2017], desenvolvido na UFRGS, é uma ferramenta para conferência entre médicos com transmissão de imagem e som. O sistema se adapta, de forma modular, automaticamente ao número de televisões na sala. Dessa forma, é possível ter um painel com quatro ou oito televisões. Além disso, é possível a integração também com pessoas que possuem um notebook, ou mesmo um dispositivo celular, onde é feita a escolha do sinal que se quer visualizar. $\mathrm{O}$ controle do sistema é feito por um tablet ou um navegador Web o qual facilita a operação, minimizando a necessidade de suporte técnico.

\footnotetext{
${ }^{1}$ Mais informações sobre a ferramenta, com sua documentação e vídeo explicativo podem ser encontradas em www.midiacom.uff.br/sthuff.
} 
O sistema de Multipresença é uma inspiração para o desenvolvimento da nossa aplicação, já que o mesmo se tornará interface do ambiente RUTE [Roesler et al. 2015] [Roesler and Harzheim 2017]. Por sua vez, tal ferramenta possui um ambiente com diversas telas e interfaces de comunicação integradas para proporcionar aos profissionais de saúde a sensação interação e participação em telecirurgias, teleconsultorias, Teleeducação, entre outros [Roesler and Harzheim 2017]. O sistema proposto funciona em tempo real para teleassistência em saúde, por multimeios em tecnologia, que permite a interação dos profissionais de saúde dentro do território nacional [Fonseca et al. 2017]. Diferentemente do sistema Multipresença, que é proposto para uso em uma infraestrutura de rede que utiliza muita banda base, devido a qualidade de vídeo e muitas telas que o ambiente propõe [Roesler et al. 2015], o sistema proposto é adequado para uso em uma banda baixa. Além disso, o custo de implantação do consultório remoto é mais barato, estima-se que o ambiente CSV custa por volta de $\mathrm{R} \$ 10.000$ [FAPERJ 2017], porém com um custo de R $\$ 2.000$ já pode ser implantado um CSV. O sistema proposto também é adaptado para o ambiente de holografia, permitindo o ajuste do som e da imagem para esse ambiente específico.

\section{Ferramenta Proposta}

A ferramenta proposta procura criar uma interface de controle para o Sistema de Saúde Holográfico da UFF que disponibilize, de forma amigável, um painel de controle para inicializar chamadas, ajustar parâmetros de multimídia, e que permita o controle de dispositivos (conteúdo) pelo próprio profissional da saúde, considerando questão de segurança e controle de acesso.

\subsubsection{Projeto de infraestrutura}

O SSH-UFF utiliza a infraestrutura mostrada na Figura 1. Esses equipamentos foram propostos para permitir uma comunicação segura e transparente. Assim, a ferramenta desenvolvida atua sobre roteadores utilizando OpenWRT para a criação do canal de comunicação seguro, além de adaptar a qualidade do vídeo à banda da conexão disponível, buscando um serviço ininterrupto e com a melhor qualidade de som e imagem possíveis.

A VPN entre os roteadores é utilizada exclusivamente para a transmissão da consulta ponto a ponto. Além disso, o sistema conta ainda com uma VPN de controle, para acesso remoto às máquinas no CSV e no CSH para ajuste manual dos parâmetros de som, imagem e vídeo.

Ainda sobre o hardware disponível, é necessário permitir a inclusão de equipamentos e dispositivos para auxiliar nos diagnósticos com a junta médica especializada. Por exemplo, faz-se o uso de um celular para transmissão de imagens da pele do paciente para auxiliar em exames dermatológicos ou transmissão de imagens do globo ocular para auxiliar em exames oftalmológicos.

O ambiente foi projetado para uso de pouca banda de transmissão, com taxa entre $1 \mathrm{Mbps}$ e 2 Mbps. Levando em conta que o CSV fica em locais remotos, a velocidade upload deve estar dentro desta faixa. 


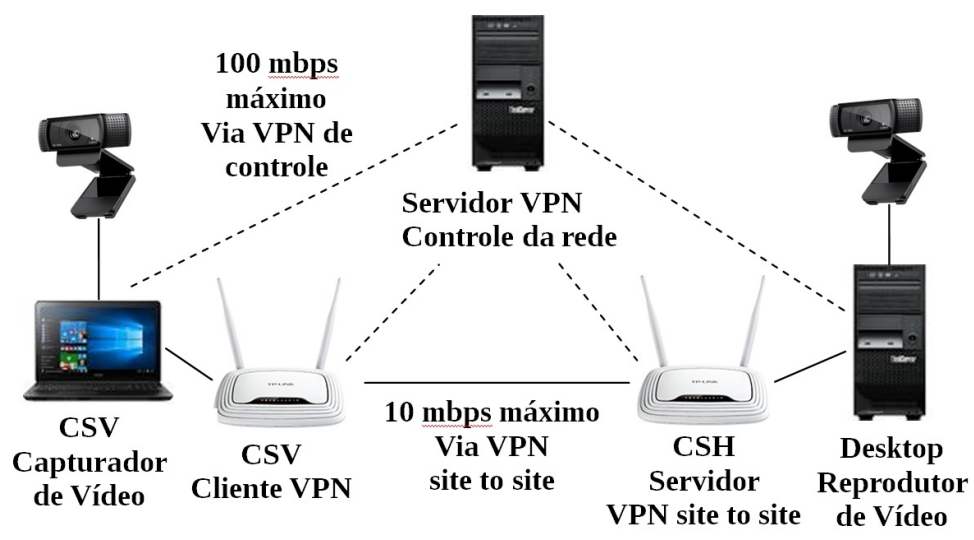

Figura 1. Infraestrutura de hardware utilizada no projeto.

\subsubsection{Arquitetura de Software}

A Figura 2 mostra a estrutura da ferramenta proposta. A ferramenta proposta é baseada no Gstreamer [Fonseca et al. 2017], mas dá suporte modular a outras formas de transmissão. Desde 2016, o SSH-UFF possui interação manual de comunicação via streaming de áudio e vídeo utilizado para comunicação entre o CSV e CSH [FAPERJ 2017]. Originalmente, existia a necessidade de suporte técnico para auxiliar os médicos durante teleinterconsulta. Assim, era necessário uma ferramenta que tornasse o ambiente amigável, deixando o profissional de saúde com a liberdade de interação sem a necessidade do suporte técnico.

A ferramenta proposta foi implementada na Linguagem Python 3 e utilizado junto com biblioteca gráfica GTK3, usada no ambiente GNOME, para criação da interface gráfica, integrada com Gstreamer e o Python. A transmissão do streaming ponto a ponto de áudio e vídeo é feita pelo serviço Real Time Streaming Protocol (RTSP) do Gstreamer, a qual permite distribuir a informação multimídia, identificando problemas de conexões em tempo de execução. Alguns exemplos de telas do aplicativo são mostrados na Figura 3. A ferramenta proposta é divida em módulos (Figura 2) descritos abaixo:

- Video Player: Responsável por integrar toda parte multimídia do sistema, nele é feita a interação entre os ambientes CSV e CSH. O mesmo integra o subsistema para controle multimídia de áudio, vídeo e ajuste de imagem para o ambiente de holografia.

- Controle Multimídia: subsistema acoplado ao modulo Video Player, nele é realizado as alterações de volume, mudança na qualidade do vídeo, além do ajuste da imagem para projeção holográfica.

- Serviço de Gravação: Esse módulo realiza toda a gravação e armazenamento da videoconferência realizada pelo Video Player. O mesmo deixa a gravação disponível somente para o(s) medico(s) e paciente(s), de cada reunião em específico.

- Controle de Acesso: Todos os usuários precisam ter um cadastro dentro do sistema, esse módulo gerencia o acesso realizado pelos participantes do telessaúde para os ambientes de CSV e CSH.

- Serviço de Chamada: Responsável iniciar uma videochamada em cada reunião. Portanto, esse módulo realiza o primeiro contato entre os ambientes CSV e CSH.

- Serviço de Agendamento: Responsável por agendar cada reunião do sistema, 


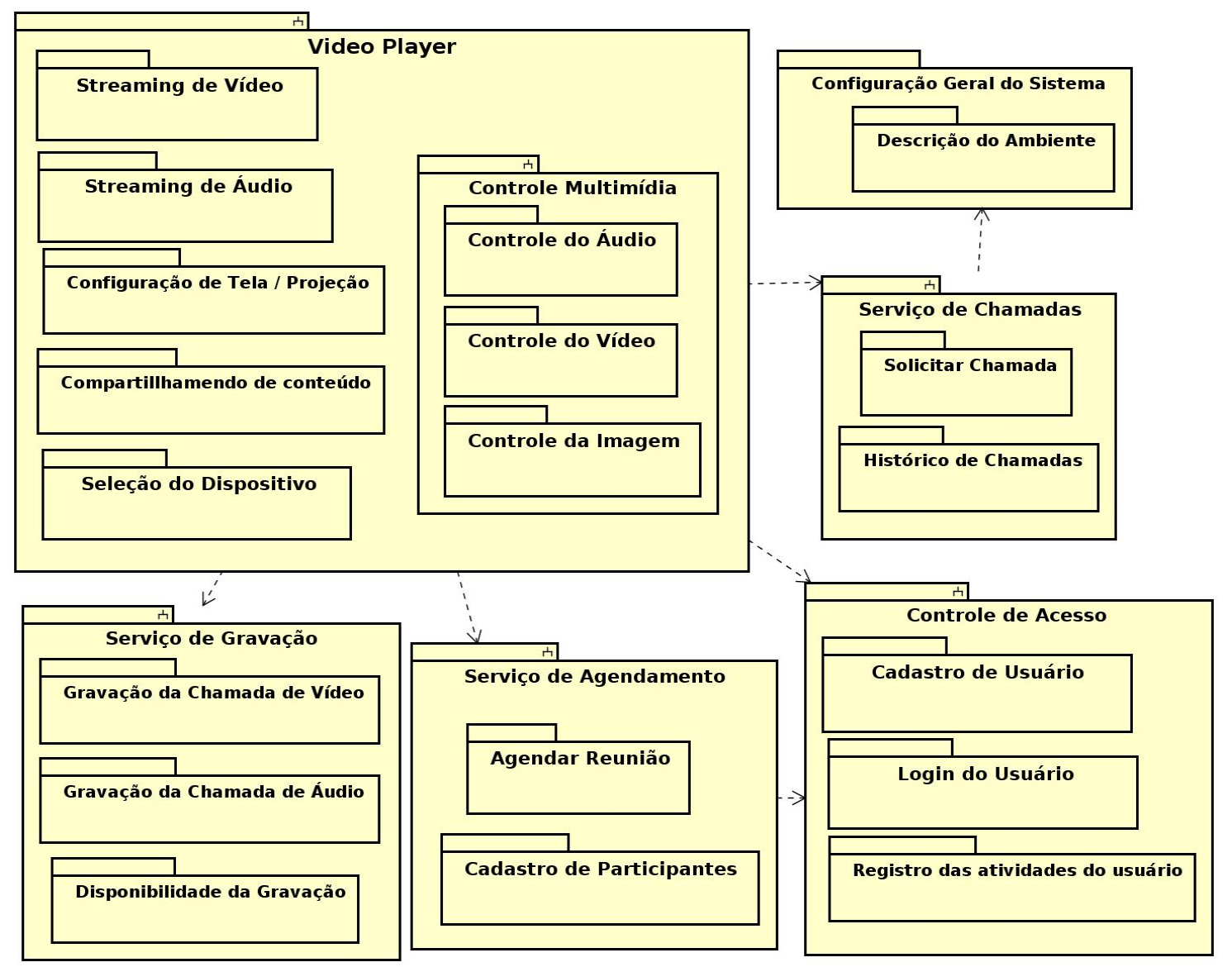

Figura 2. Módulos da ferramenta proposta.

associando a ela os participantes: médico(s) e paciente(s). Cada reunião terá informações sobre o quadro clínico do paciente e a especialidade médica do atendimento.

- Configuração Geral do Sistema: Módulo com mais detalhes do ambiente cadastrado, entre outras configurações adicionais do sistema.

\section{Demonstração}

As funcionalidades da ferramenta podem ser demonstradas em qualquer local que possua dois computadores com acesso à Internet, um representando o CSV e o outro, o CSH. Para a demonstração, serão levados dois roteadores com OpenWRT, duas webcams e um celular com Android. Utilizando os computadores no local, será feito o uso da ferramenta desenvolvida para ajustar a imagem e o som, iniciar e gravar uma consulta, e mostrar o uso de imagens auxiliares para diagnóstico. Além disso, a qualidade do enlace entre os computadores será modificada, mostrando como o sistema reage à qualidade da rede ${ }^{2}$.

\section{Conclusão}

A ferramenta proposta foi desenvolvida especificamente para uso em telessaúde, permitindo uma projeção holográfica em tempo real (única no brasil), com ajustes multimídia,

\footnotetext{
${ }^{2}$ Caso seja de interesse da organização, poderá ser agendada uma ou mais demonstrações no CSH e no CSV da UFF, emulando consultas reais com holografia
} 


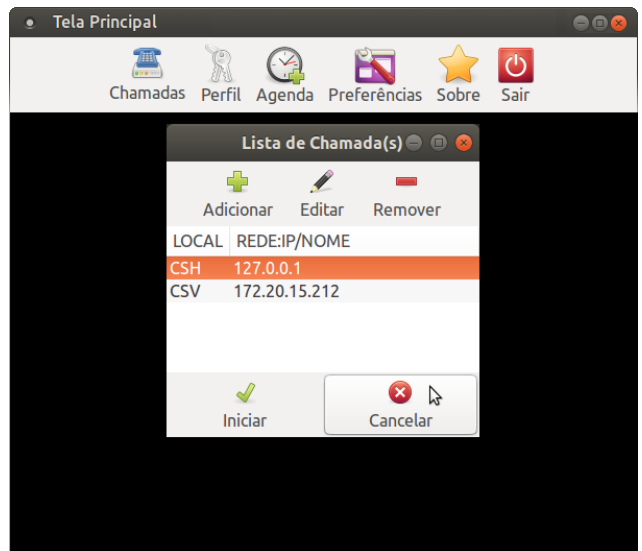

(a) Tela Principal, aonde o médico escolhe com qual sala se conectar.

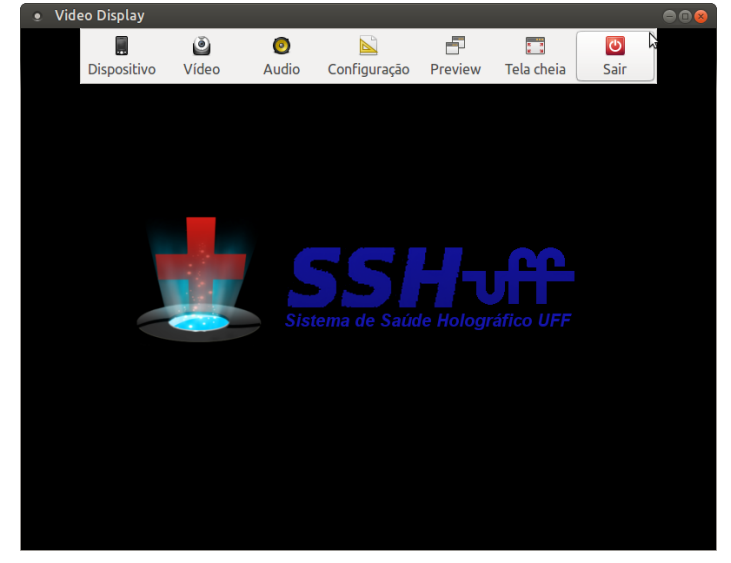

(b) Tela de reprodução do streaming, que permite a configuração de mídia para permitir uma melhor imagem holográfica e melhor som.

Figura 3. Telas da ferramenta proposta

levando em conta a comunicação segura e considerando que apenas os médicos cadastrados podem se autenticar e acessar o sistema. Além disso, a ferramenta disponibiliza as gravações das videochamadas de forma secura e acessível apenas para os participante(s) de cada reunião agendada. A ferramenta se mostrou apta para auxiliar a teleinterconsulta, ampliando visões adicionais para diferentes tipos de especialidades médicas.

\section{Bibliografia}

Dal Bello, J. C. R. (2016). Implantação e execução de sistema holográfico em centro de saúde da uff vinculado a ações de saúde com a marinha do brasil (projeto telessaúde). Technical report, Universidade Federal Fluminense, Niterói - RJ. Apoio com FAPERJ.

FAPERJ (2017). Saúde ao vivo, a cores e em 3D. Rio Pesquisa, (38):26-28. Ano IX.

Fonseca, A. L. d. O., Beaklini, A. C., Vale, E. R., Filho, R. P., Dal Bello, J. C. R., Boechat, Y. E., Carrano, R. C., and Fernandes, N. C. (2017). Interiorização da medicina utilizando um Sistema de Telepresença Holográfico. In Anais do XXIII Simpósio Brasileiro de Sistemas Multimídia e Web: Workshop do CT-Vídeo, Gramado, Rio Grande do Sul - RS. Sociedade Brasileira de Computação (SBC).

Roesler, V. and Harzheim, E. (2017). Tecnologias emergentes em telemedicina - ênfase em videocolaboração. In Anais do XXIII Simpósio Brasileiro de Sistemas Multimídia e Web: Workshop do CT-Vídeo, Gramado, Rio Grande do Sul - RS. Sociedade Brasileira de Computação (SBC).

Roesler, V., Longoni, G., Marins, A., et al. (2015). Multipresença: um sistema de videoconferência adaptável, escalável e interoperável.

RUTE (2019). Rede universitária de telemedicina (rute). Disponível em: rute.rnp. br. Acessado em: 2019-03-01.

Scheffer, M., Cassenote, A., Guilloux, A. G. A., Biancarelli, A., Miotto, B. A., and Mainardi, G. M. (2018). Demografia Médica no Brasil 2018. Conselho Regional de Medicina do Estado de São Paulo, Conselho Federal de Medicina, São Paulo - SP. Departamento de Medicina Preventiva, Faculdade de Medicina da USP. 